\title{
Sclerostin: Intracellular mechanisms of action and its role in the pathogenesis of skeletal and vascular disorders
}

\author{
Barbara Pietrzyk ${ }^{\mathrm{D}}$, Mike Smertka ${ }^{\mathrm{D}}$, Jerzy Chudek $^{\mathrm{D}-\mathrm{F}}$ \\ Department of Pathophysiology, Medical University of Silesia, Katowice, Poland \\ A - research concept and design; B - collection and/or assembly of data; $\mathrm{C}$ - data analysis and interpretation; \\ $\mathrm{D}$ - writing the article; $\mathrm{E}$ - critical revision of the article; $\mathrm{F}$ - final approval of the article
}

\author{
Address for correspondence \\ Jerzy Chudek \\ E-mail: chj@poczta.fm \\ Funding sources \\ Grant from Medical University of Silesia in Katowice \\ (KNW-1-004/N/7/0)
}

\section{Conflict of interest}

None declared

Received on November 24, 2015

Reviewed on May 27, 2016

Accepted on January 31,2017

\begin{abstract}
Sclerostin is a glycoprotein involved in the regulation of bone metabolism, exclusively secreted by osteocytes. It affects the activity of bone morphogenetic proteins (BMPs) and is an inhibitor of the Wnt/B-catenin metabolic pathway in bone cells. Osteocytes reduce the release of sclerostin in response to mechanical stimuli acting on bone, and thus promote the activation of osteogenic pathway Wnt/B-catenin in osteoblasts. This signaling pathway plays a key role in osteogenesis and bone turnover. Loss of sclerostin gene function is related to 3 different craniotubular hyperostosis processes: sclerosteosis, craniodiaphyseal dysplasia, and van Buchem disease. Additionally, experimental and clinical studies suggest that sclerostin may promote vascular calcification. Antibodies directed against sclerostin stimulate bone formation and represent a new therapeutic option in the treatment of diseases with increased bone resorption, such as osteoporosis and inflammatory diseases where there is generalized bone loss, periarticular osteoporosis, and cartilage damage, such as rheumatoid arthritis (RA), ankylosing spondylitis (AS), and glucocorticoid-induced osteoporosis (GIO). Antibody use has the potential to offer new therapeutic approaches in the therapy of mineral and bone disorders resulting from chronic kidney disease (CKD-MBD) and vascular calcifications.
\end{abstract}

Key words: osteoporosis, bone mineral density, vascular damage, sclerostin, Wnt/B-catenin pathway

DOI

$10.17219 /$ acem $/ 68739$

Copyright

Copyright by Author(s)

This is an article distributed under the terms of the

Creative Commons Attribution Non-Commercial License

(http://creativecommons.org/licenses/by-nc-nd/4.0/) 


\section{Introduction}

Bone cells include osteocytes, osteoblasts, and osteoclasts with each having a different and specific roles in bone remodeling, and are essential to the maintenance of biomechanical functions of the skeleton. Physiological bone remodeling involves the continuous process of bone destruction, resorption, and formation, resulting in the production of osteoid (early phase) and its mineralization (late phase). A key role in this process is played by the basic multicellular unit (BMUs), which is a temporary anatomic structure. The opposing processes of bone remodeling are separated by a rest phase, when the surface of newly formed bone is covered by osteocytes. Apoptosis of osteocytes characterize the transition from the resting phase to the resorption phase of the bone turnover.

Osteocytes buried in the bone matrix act as mechanosensors, specializing in the receiving and transmission of signals related to mechanical stress, microtrauma, and microfractures, which play a primary role in the stimulation process of bone formation. ${ }^{1}$ Sclerostin plays an important role in osteocyte-osteoblast signaling.

\section{The role of sclerostin in the regulation of bone turnover}

Osteocytes are the bone cells with the longest longevity; they constitute $90-95 \%$ of all cells in human bone. ${ }^{2}$ Their role in bone formation is related to recruitment of pre-osteoblast precursors derived from mesenchymal stem cells by stimulation of the sclerostin-Wnt (Wingless-type signaling) signaling pathway. ${ }^{3}$ Mature osteoblasts regulate the bone remodeling processes through the production of receptor activator for nuclear factor $\mathrm{\kappa} B$ ligand (RANKL), regulated by $17 \beta$-estradiol and parathyroid hormone $(\mathrm{PTH})$ though receptors which include parathyroid hormone receptor type 1 (PTHR1) and estrogen receptor $\beta$ (ER $\beta$ ).

Osteoblasts produce osteoid, bone matrix, and osteocalcin, which is a specific marker of osteoblast activity. When embedded in the bone matrix, osteoblasts undergo transformation into osteocytes, and then increase the expression of such particles such as denin matrix protein-1 (DMP1) and sclerostin. ${ }^{4}$ Sclerostin is formed by osteocytes and transferred by cytoplasmic extensions to osteoblasts, which are located on the surface of bone trabeculae. ${ }^{5}$ In response to mechanical loads, osteocytes secrete sclerostin through the modulation of the transforming growth factor- $\beta$ (TGF- $\beta$ ) dependent pathway. ${ }^{6}$

Sclerostin is a key molecule that inhibits osteoblast activity (Fig. 1). It binds mostly to low-density lipoprotein receptor-related protein 5/6 (LPR5/6) and facilitates intracellular actions. ${ }^{6,7}$ Activation of the Wnt/ $\beta$-catenin pathway promotes osteoblastogenesis by stimulating osteoblast differentiation and inhibiting osteoclastogenesis by the RANK/ RANKL signaling pathway. ${ }^{8}$ According to a new concept, mechanical loads not only directly stimulate osteoblasts but mainly decrease the synthesis of SMAD, a group of cytoplasmic signaling proteins in osteocytes that are necessary for the upregulation of SOST gene expression.

As a consequence, sclerostin production is decreased, which indirectly stimulates osteogenesis. ${ }^{9}$ Sclerostin can antagonize BMP signaling directly by inhibiting BMP7 secretion. Sclerostin interacts with both the BMP7 proand mature domains, and leads to intracellular retention and proteasomal degradation of BMP7. Sclerostin exerts its potent catabolic effects on bone by antagonizing Wnt signaling in a paracrine and autocrine manner, as well as antagonizing BMP signaling selectively in the osteocytes that simultaneously synthesize both sclerostin and BMP7 proteins. Wnt proteins are a family of glycoproteins that assist in triggering many signal pathways, which are important for the differentiation and regeneration of tissue. ${ }^{10}$ Sclerostin is a Wnt-dependent protein that has a potent inhibitory effect on the Wnt/ $\beta$-catenin pathway, stimulating osteogenesis by promoting the differentiation and proliferation of osteoblasts, inhibition of apoptosis and enhancement of osteoid mineralization. ${ }^{11}$ The signals conduction on the Wnt pathway represents a critical point for bone metabolism. ${ }^{12}$

Wnt/B-catenin signaling is fundamental for osteoblast differentiation, by stimulating (RUNX2, DLX5, MSX2, $O S X)$ or inhibiting (C / EBP- $\beta$ / $\delta, C / E B P-\alpha$, PPAR- $\gamma$ ) the respective genes and related proteins; blocking development and differentiation of mesenchymal stromal cells of the adipogenesis and chondrogenesis pathway. ${ }^{13}$ The control of the Wnt/3-catenin pathway is regulated by transcription factor Osterix (OSX), which is highly specific for osteoblasts. ${ }^{13}$

OSX factor is a target for sclerostin, which activates the promoter SOST and encodes sclerostin, which in turn inhibits Wnt signaling. ${ }^{11}$ Sclerostin facilitates osteoblastic cell apoptosis through the activation of caspases. Wnt proteins are a family of ligand-receptor Frizzled (Fzd) and are used to initiate the conduction of intracellular signaling pathways, resulting in the stabilization of $\beta$-catenin, the main transducer in the Wnt signaling pathway. ${ }^{14}$

$\beta$-catenin increases synthesis of osteoprotegerin (OPG), and the ratio of RANKL to OPG, inhibits osteoclastogenesis. ${ }^{15}$ Serum proteins, sclerostin and Dickkopf-1 (Dkk-1), act as soluble inhibitors of the canonical Wnt signaling pathway, which has recently been identified as the signaling pathway in PTH transduction. ${ }^{16}$

The canonical Wnt signaling path includes the formation of complexes of Wnt with frizzled receptors, as well as with co-receptor LRP5/6, resulting in the accumulation of cytoplasmic $\beta$-catenin and its translocation to the nucleus. ${ }^{17}$ The interaction of inhibitors (e.g., sclerostin) on the frizzled-glomerulus receptor and co-receptor LRP5/6, which binds to the Wnt protein signal, determines whether intracellular concentration of $\beta$-catenin will be high enough in the cell nucleus to cause a biological effect (stimulation of osteogenesis). ${ }^{15}$ 
Dkk-1 inhibits Wnt signaling by forming a complex with LRP5/6 and silicon, resulting in the removal of LRP $5 / 6$ from the cell membrane. Antagonistic activity of the sclerostin-Wnt pathway requires silicon as a cofactor, which in turn antagonizes Dkk-1. This is a subject of research. ${ }^{17}$ Also, as sclerostin increases mRNA expression of RANKL, this in turn activates nuclear factor $\mathrm{kB}$, which further activates genes required for osteoclast differentiation. ${ }^{18}$ In the RANK/Osteoprotegerin/RANKL system, sclerostin decreases mRNA for OPG, which promotes osteoclastogenesis and bone resorption. ${ }^{19}$

As previously described, intracellular signal transduction is responsible for the control of osteoblast development, proliferation, differentiation, migration, and apoptosis. ${ }^{20}$ In healthy bone, osteocytes maintain a balance between osteolysis and osteogenesis through the control of sclerostin secretion. The effect of mechanical forces and microtrauma of bone inhibit the secretion of sclerostin by osteocytes, intensifying the process of bone formation. ${ }^{21}$

\section{Diseases related to genetic disorders of sclerostin}

In humans, sclerostin is encoded by the SOST gene located on chromosome 17q12-q21. Loss of sclerostin function was shown as the cause of massive amounts of normal bone formation throughout life, due to the absence of an efficient mechanism for the fine-tuning of BMP activity and bone formation..$^{22,23}$

There are 3 different craniotubular hyperostoses related to the loss of SOST function. The first is sclerosteosis, which is a homozygous SOST mutation (MIM 269500). The second, craniodiaphyseal dysplasia (CDD), is caused by a heterozygous SOST mutation (MIM 122860). Finally, van Buchem disease is caused by a $52-\mathrm{kb}$ deletion approx. $35 \mathrm{~kb}$ downstream of SOST that removes a SOST-specific regulatory element (MIM 239100).

The concept of genotype-phenotype correlation in this group of disorders is tenable. Sclerosteosis 1 and van $\mathrm{Bu}$ chem disease are clinically and radiographically similar disorders. ${ }^{22}$ These disorders include osteosclerosis, notable osteopetrosis, which is characterized by increased bone density, with no bone overgrowth, and little or no disturbance of bones contours. Radiographic findings include widening (hyperostosis) of the calvarium, the base of the skull, and the shafts of tubular bones. SOST is the only gene known to be associated with sclerosteosis and van Buchem disease. CDD is characterized by abnormal modeling of the skeleton and moderate sclerosis of the calvarium and base of the skull. The manifestations of van Buchem disease are generally milder than those of sclerosteosis and syndactyly is absent. The spectrum of SOST-related sclerotizing bone dysplasias includes an autosomal dominant form of CDD. ${ }^{24}$ In contrast, van Buchem disease is an autosomal recessive bone dyspla- sia characterized by a symmetrically increased thickness of bones, most frequently found as an enlarged jawbone, but also as an enlargement of the skull, ribs, diaphysis of long bones, as well as tubular bones of hands and feet, resulting in increased cortical bone density. The clinical consequences of the increased thickness of the skull include facial nerve palsy resulting in hearing loss, visual problems, neurologic pain, and very rarely, blindness resulting from optic atrophy. Bone anomalies appear in the first decade of life and progress with age. ${ }^{25}$

Sclerosteosis 1 is an uncommon, autosomal recessive, progressive sclerosing bone dysplasia characterized by generalized osteosclerosis and hyperostosis of the skeleton, primarily affecting the skull, mandible, and diaphyseal regions of the long bones. In most patients, skull changes cause facial paralysis and hearing loss. Other features are gigantism and hand abnormalities. Carriers (heterozygotes) of pathogenic SOST variants are not affected by any clinical symptoms of sclerosteosis, but they undergo the process of bone turnover and exhibit bone mineral density at a level that is at an intermediate level between normal and affected individuals, demonstrating the body's exquisite sensitivity to absolute levels of sclerostin. ${ }^{26}$ In CDD, the main phenotypic features are progressive overgrowth of the craniofacial bones with deafness, facial palsy, and visual disturbance as a result of nerve entrapment. Radiologically, the cranial and facial bones are hyperostotic, while the diaphyses of the limb bones are expanded with thin cortices. ${ }^{24}$ Heterozygous SOST mutations have been documented also in CDD affected individuals in Poland. ${ }^{27}$ Apart from sclerosteosis 1, van Buchem disease, and $\mathrm{CDD}$, no other simple genetic disorders are known to be associated with mutations in SOST. However, phenotypically similar sclerosteosis 2 may be caused by a mutation of LRP4 (low density lipoprotein receptor-related protein 4) that is involved in sclerostin signal transduction (MIM 614305). Additionally, common non-coding polymorphisms in SOST were found to moderately affect bone mineral density in the elderly, suggesting a role for varied SOST expression in osteoporosis. ${ }^{28}$ As is the case with parathyroid hormone having anabolic properties, and hence excessive sclerostin-induced activation of the actuating mechanism of cell proliferation by Wnt, it may also initiate the process of tumorigenesis, which involves the risk of osteogenic sarcoma (osteosarcoma). ${ }^{29}$

\section{Circulating sclerostin levels in relation to age, gender, menopausal status, bone mineral density, and postmenopausal osteoporosis}

As was already mentioned, sclerostin antagonizes canonical Wnt signaling by binding to Wnt co-receptors, LPR4, LPR5 and LPR5/6. The ineffective inhibition of Wnt 
signaling may be caused by decreased production of sclerostin (sclerosteosis 1, CDD, van Buchem disease) or defective sclerostin binding to LPR4 (sclerosteosis 2). Aside from the group of rare genetic diseases, the observable variability of serum sclerostin levels affects BMD of both cortical and cancellous bone in the general population. The molecular effect of sclerostin levels and BMD were studied by Reppe et al. ${ }^{30}$ They showed that the genetic and epigenetic changes in SOST influence its mRNA expression in bone and serum sclerostin levels in postmenopausal women. Serum sclerostin and bone SOST mRNA expression correlated positively with age-adjusted and BMI-adjusted total hip BMD ( $\mathrm{r}=0.47)$ and inversely to serum bone turnover markers. The observations suggest that increased SOST promoter methylation, seen in osteoporosis, is a compensatory, counteracting, mechanism which lowers the production of sclerostin and reduces the inhibition of Wnt signaling in an attempt to promote bone formation. ${ }^{30}$

Mödder et al. measured serum sclerostin levels in a population-based sample of 362 women: 123 premenopausal, 152 postmenopausal not on estrogen treatment (ET), and 87 postmenopausal on ET; and 318 men, aged 21-97 years. Sclerostin levels were significantly higher in men than in women, and increased markedly with age. When compared to younger subjects, elderly individuals had higher serum sclerostin levels for a given amount of bone mass. It has been suggested that circulating sclerostin levels might reflect total-body skeletal mass, the larger skeletal mass in men may simply produce and release more sclerostin into circulation. They also found that among postmenopausal women, serum sclerostin levels were significantly lower in women on ET compared with untreated women. Sclerostin levels were significantly associated with age in the combined group of pre- and postmenopausal women not on ET $(r=0.52)$ and in men $(r=0.64) .{ }^{31}$

Estrogen signaling in osteoblasts negatively regulates SOST expression in an indirect manner through interaction with BMP2 signaling. This regulation involves the Wnt/ER $\alpha$ and $\beta$-catenin pathways. ${ }^{32}$ Mirza et al. compared serum sclerostin levels in premenopausal (mean age 26.8 years) and postmenopausal (mean age 56.8 years) women and evaluated its relationship to estrogen, PTH, bone turnover, and bone mass. Postmenopausal women had lower values of estradiol and free estrogen index (FEI), and significantly lower bone mineral density at all sites compared to premenopausal women; with no significant differences in PTH, 25-hydroxy or 1,25-dihydroxy vitamin D levels. Postmenopausal women had significantly higher serum sclerostin levels $(1.16 \pm 0.38$ vs $0.48 \pm 0.15 \mathrm{ng} / \mathrm{mL}$ ). There were significant negative correlations between sclerostin and FEI and PTH in this group. Using multiple regression analysis, both FEI and PTH were found to be independent predictors of sclerostin levels in postmenopausal women. These findings suggest that serum sclerostin levels are regulated by both estrogens and $\mathrm{PTH} .{ }^{33}$ Among other factors affecting sclerostin levels, vitamin D should be mentioned. It was shown that a supraphysiological concentration of 1,25-dihydroxy vitamin $\mathrm{D}$, following a loading dose of vitamin D, increases sclerostin and may inhibit Wnt signaling. This may have unexpectedly detrimental effects on the bone. ${ }^{34}$ Increased sclerostin levels and reduced bone mineral density have been documented in adult patients with diabetes, predominantly in those with type 2 diabetes (T2D). Circulating biochemical markers of bone formation, including P1NP, osteocalcin, and bone-specific alkaline phosphatase, have been found to be decreased in T2D and may be predictive for fractures independently of bone mineral density (BMD). Serum sclerostin levels have been found to be increased in T2D and appear to predict fracture risk independent of BMD. ${ }^{35}$

Sclerostin levels and changes in bone metabolism have also been studied after bariatric surgery. Muschitz et al. described an increase in serum sclerostin that was positively correlated with CTX and P1NP increases but inversely correlated with BMD loss. Elevations of sclerostin, CTX, P1NP, but not DKK-1 and iPTH, were significant discriminating factors for BMD loss post procedure. ${ }^{36}$

Osteoporosis is a systemic skeletal disease. This disease is characterized by low bone mineral density and microarchitecture weakening of the bone tissue, and in consequence an increase in bone fragility and susceptibility to low-trauma fractures. Post-menopausal women are predisposed to osteoporosis development due to estrogen deficiency affecting bone remodeling. Deficiency of vitamin D and calcium leads to impaired bone deposition and increases bone resorption through increased secretion of parathyroid hormone (PTH) by parathyroid glands that react to low calcium levels. ${ }^{37}$

In postmenopausal osteoporosis estrogens deficiency enhance bone resorption. Osteocytes are not only the major source of sclerostin, bone formation inhibitor, but also the source of receptor activator of NF-kB ligand (RANKL), an essential cytokine responsible for osteoclast activation and bone resorption in postmenopausal osteoporosis. In addition, there is some evidence for the role of abnormal sclerostin signaling in postmenopausal women. Recently increased methylation of SOST promoter was found in bone biopsies of postmenopausal women. It is considered as a compensatory, counteracting mechanism, which lowers sclerostin production and reduces inhibition of Wnt signaling in an attempt to promote bone formation. ${ }^{38}$ However, serum sclerostin is unaffected by menopausal status or increase in age, perhaps due to kidney aging accompanied by deterioration of their excretory and biodegradation functions. It is generally accepted that sclerostin in postmenopausal women reflects bone mineral density. ${ }^{39-41}$

Morales-Santana et al. showed the association between circulating levels of sclerostin and estradiol with an inadequate clinical outcome to bisphosphonate therapy in postmenopausal women with osteoporosis. Sclerostin 
and estradiol levels were associated with the response to bisphosphonate therapy in women with postmenopausal osteoporosis. Also, sclerostin concentrations were associated with the index of resistance to compression (IRC) in the fractal analysis of the distal radius, a parameter of bone microstructure. ${ }^{42}$

\section{Inflammatory diseases related to disorders of sclerostin}

Rheumatoid arthritis (RA) and ankylosing spondylitis (AS) are 2 inflammatory joint diseases characterized by bone complications including osteoporosis. Bone loss, bone erosion, and systemic osteoporosis with an increased risk of fractures are observed periarticularly in RA. Determinants of fractures are underlying conditions, severity of the disease, use of glucocorticoids and reduced physical activity. Prospective data showed that the optimal control of inflammation in RA is associated with a decrease in structural damage and bone loss. RA illustrates the role of inflammation on bone resorption. In AS, osteoporosis is an early event, related to inflammation and glucocorticoids use, increasing the risk for vertebral fractures. AS is characterized by progressive stiffness and ankylosis of the spine and illustrates also the potential role of inflammation on local bone formation. ${ }^{43}$ Osteoclastogenesis is under the control of RANKligand, which is produced by osteocytes in normal bone remodeling, but also by lymphocytes and fibroblasts in inflammation. ${ }^{44}$ Osteoclastogenesis can be stimulated by a number of cytokines, the main pathway by Th 17 cells subpopulation interleukin (IL) IL-6 and IL-23. ${ }^{45}$ A major role in bone remodeling process is played by autoimmunity, as antibodies against citrullinated proteins (ACPAs) can increase osteoclast numbers and their activity through citrullinated vimentin located at the surface of precursors and cells (through a TNF- $\alpha$ local effect). ${ }^{46}$ Tumor necrosis factor $\alpha$ (TNF- $\left.\alpha\right)$ transgenic mice are models of osteoporosis with a dramatic decrease in bone mass and impairment of bone microarchitecture. Overexpression of sclerostin with inflammation-related decrease in bone formation has been observed in animal models. Therefore, sclerostin inhibition is considered as a potential, powerful tool to enhance bone repair in inflammatory arthritis. ${ }^{47}$ In a research study on an experimental model in mice, Yao et al. showed that treatment with monoclonal antibody against sclerostin (Scl-Ab) prevented glucocorticoid (GC)-induced reduction in both trabecular and cortical bone mass and strength, and appeared to maintain osteoblast activity through autophagy. ${ }^{48}$ Glucocorticoids increase the expression of Wnt signaling antagonists (sclerostin and Dkk-1) in experimental studies on rodents. In humans, especially in young glucocorticoid-induced osteoporosis (GIO), the most common form of secondary osteoporosis and the first iatrogenic cause of disease are observed. In the pathogenesis of GIO, the main feature is the suppression of bone formation, decreasing the replication of cells of the osteoblastic line, as well as their differentiation and maturation. GIOP disrupts the normal function of osteoblasts by interfering with the signal Wnt/ $\beta$-catenin pathway, which is the most important element of regulating the life of osteoblasts. This pathway is inhibited by Wnt-antagonists sclerostin and Dkk-1. Animal and in vitro studies have shown an over-expression of these antagonists with glucocorticoid therapy. In humans contradictory results have been shown, although most point to an increase in sclerostin serum levels, probably due to the dose and duration of treatment. ${ }^{49}$ Bone loss and increased rate of fractures occur early after the initiation of corticosteroid therapy, and are then related to dosage and treatment duration. Bone fragility in GIOP is characterized by the rapidity of bone loss at the introduction of GCs, and the discrepancy between bone mineral density (BMD) and risk of fractures. The increase of fracture risk is not fully assessable by bone mineral density measurements, as it is also related to the alteration of bone quality and increased risk of falls. ${ }^{50}$

\section{Circulating sclerostin levels and calcifications}

There is a hypothesis that sclerostin might also play a role in the development of aortic valve and vascular calcifications. Koos et al. evaluated serum sclerostin levels in patients with different degrees of aortic valve calcification (AVC) compared to healthy controls and investigated local sclerostin expression in explanted calcified and non-calcified aortic valves. Patients with AVC showed increased sclerostin serum levels and revealed that the severity of AVC is proportional to sclerostin serum levels. Moreover, the increased sclerostin expression was parallel to prototypic markers of osteogenic transdifferentiation, indicating the role of sclerostin in the valvular calcification process. ${ }^{51}$ A study conducted by Hampson et al. confirms this hypothesis. They investigated the association between circulating concentrations of Wnt inhibitors, Dkk-1 and sclerostin with bone mineral density, abdominal aortic calcification (AAC), and arterial stiffness in post-menopausal women. A significant positive correlation was observed between sclerostin and BMD at the femoral neck $(r=0.17)$ and total hip ( $r=0.16)$. In adjusted linear regression analysis, sclerostin was positively associated with AAC. Additionally, they showed that subjects with aortic pulse wave velocity (PWV) $>9 \mathrm{~m} / \mathrm{s}$ had significantly higher serum sclerostin levels than those with PWV $<9$ m/s $(23.8$ vs 29.7 $\mathrm{pmol} / \mathrm{L}) .^{52}$ Recently, Kuipers et al. showed that increases by 1 standard deviation of sclerostin levels are associated with a 1.61-times (95\% CI 1.02-2.53) greater risk of having coronary artery calcification. ${ }^{53}$ 


\section{Sclerostin in chronic kidney disease}

Of the multiple disorders developing in patients with chronic kidney disease (CKD), of particular interest here are mineral and bone disorders coexisting with vascular calcification, called CKD-MBD (chronic kidney disease - mineral and bone disorders). This disorder enhances with a decrease in glomerular filtration, phosphate retention, and insufficient renal hydroxylation of $25-\mathrm{OH}-\mathrm{D}_{3}$, which results in reduced absorption of calcium from the gastrointestinal tract, increased secretion of PTH (secondary hyperparathyroidism) and fibroblast growth factor 23 (FGF23). ${ }^{54}$ One of the components of CKD-MBD is vascular calcification, in particular on the middle membrane of muscular arteries (Moenckeberg type) that increase arterial stiffness. This type of calcification results in the increase in systolic and decrease in diastolic blood pressure, followed by an increase in the afterload and hypertrophy of the left ventricle and the reduction of coronary blood flow in diastole. ${ }^{55}$ These changes are associated with increased cardiovascular risk. ${ }^{56}$ The main factor stimulating transformation of vascular smooth muscle cells (VSMC) to osteoblast-like cells is hyperphosphatemia. Experimental studies have shown that hyperphosphatemia in VSMC increases methylation of the DNA sequence of the promoter and hence inhibits expression of the gene transgeline $(S M 22 \alpha)$, a protein specific to muscle cells, and increase expression of the transcription factor RUNX2 (runt-related transcription factor 2). ${ }^{57}$ The increased expression and activation of RUNX2 (resulting in phosphorylation-dependent mitogen-activated protein kinase (MAPK) initiates the transformation VSMC to osteoblasts-like cells. ${ }^{58}$ The factors intensifying the process of vascular calcification probably include sclerostin and FGF-23. Experimental studies show increased expression of sclerostin in VSMC during calcification. ${ }^{59}$ In the case of FGF23, we have only indirect evidence in the form of increased cardiovascular mortality in CKD patients, including those on dialysis, with higher concentrations of FGF23 in the circulation. ${ }^{60}$ It would, therefore, be expected that higher concentrations of sclerostin, like those of FGF-23, should increase cardiovascular risk, but there is conflicting evidence. ${ }^{61}$ Some studies show no association between sclerostin and mortality. ${ }^{62}$

The decrease in GFR and concomitant reduction of sclerostin biodegradation is potentially the main cause of the increased concentrations of this protein in circulation, despite increases in the fraction excreted into urine with the progression of CKD. ${ }^{63,64}$ As with subjects having normal renal function, higher concentrations of sclerostin occurs in men, which can be explained by a higher bone mass and indirectly a greater number of osteocytes. ${ }^{65}$ The relationship between the concentration of phosphorus and sclerostin (independent from GFR) indicates its potential contribution to the regulation of its secretion by osteocytes. ${ }^{66}$ Ishimura et al. showed that serum sclerostin was significantly and independently associated with BMD. The BMD in the distal $1 / 3$ of the radius and in the ultradistal radius both correlated significantly and positively with serum sclerostin levels $(r=0.45)$. In multiple regression analysis, serum sclerostin was associated significantly and independently with BMD, after adjustment for age, hemodialysis duration, and bone metabolism markers. ${ }^{67}$ Delanaye et al. confirmed the presence of higher concentrations of sclerostin in hemodialyzed patients (HD), a positive association with age and a negative association with $\mathrm{PTH} .{ }^{62}$

Logically, the accumulation of sclerostin in circulation should lead to a stronger inhibition of osteoblasts and lower level of bone formation. However, lower bone turnover and increased bone resorption was observed. It was demonstrated that CKD-MBD is associated with a higher risk of cardiovascular disease, increased vascular calcification, and circulating levels of $\alpha$-klotho protein, FGF23, and sclerostin. Even in the early stages of CKD, a reduction in $\alpha$-klotho protein content in vessels was shown to stimulate osteoblast transition in the arterial wall, secretion of proteins by osteocytes, and to inhibit bone remodeling. ${ }^{68}$ Contrary to sclerostin, the concentration of DKK-1, an inhibitor of Wnt signaling pathway, and PTH signal transduction, was not increased in dialysis patients with end-stage renal failure (stage 5 CKD). Additionally, an inverse correlation between concentrations of iPTH and sclerostin, and between sclerostin and histomorphometric parameters of bone turnover, even after adjusting for age, gender, hemodialysis vintage, presence of diabetes, and vitamin D treatment was shown. The observed correlation between sclerostin and cancellous bone volume was lost in multiple regression analysis. ${ }^{17}$ Contrary serum Dkk-1 levels did not correlate with iPTH or with any histomorphometric parameter of bone turnover. ${ }^{17}$ According to the study of Pelletier et al., serum sclerostin variability in CKD patients was explained by GFR, sex, and serum phosphate activity in multiple regression analysis. ${ }^{16}$ These results suggest that serum phosphate may stimulate sclerostin synthesis by osteocytes, but does not uncover the potential mechanism hiding beneath and does not explain previously observed inverse relation with iPTH. ${ }^{16,17}$

\section{Antisclerostin antibodies in established and potential therapies}

Inhibition of sclerostin, a glycoprotein secreted by osteocytes, offers a new therapeutic paradigm for treatment of osteoporosis. Blosozumab treatment resulted in statis- 
Fig.1. The probable model for the mechanism of action of sclerostin on the process of bone formation

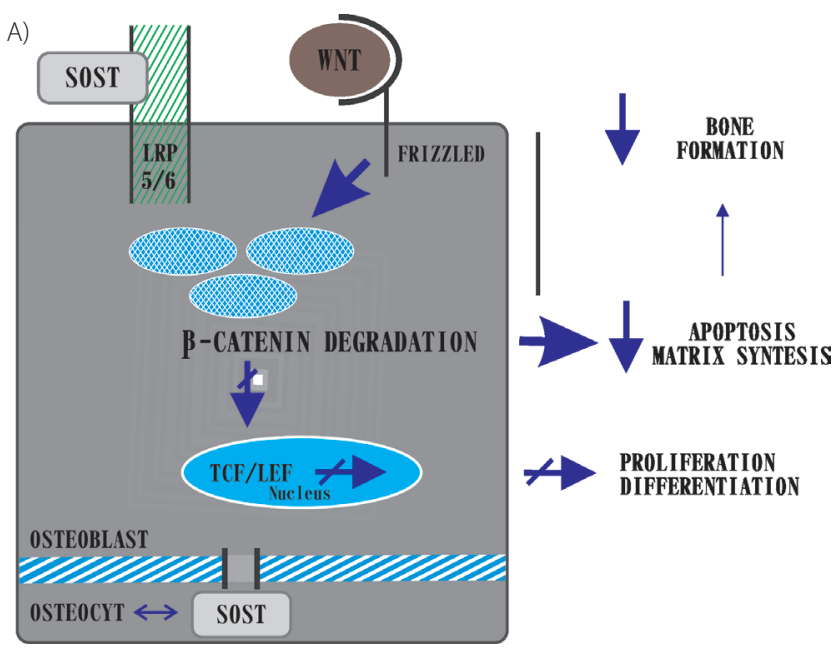

TCF/LEF - T cell factor / Lymphatic Enhancing Factor (Wnt co-receptors).

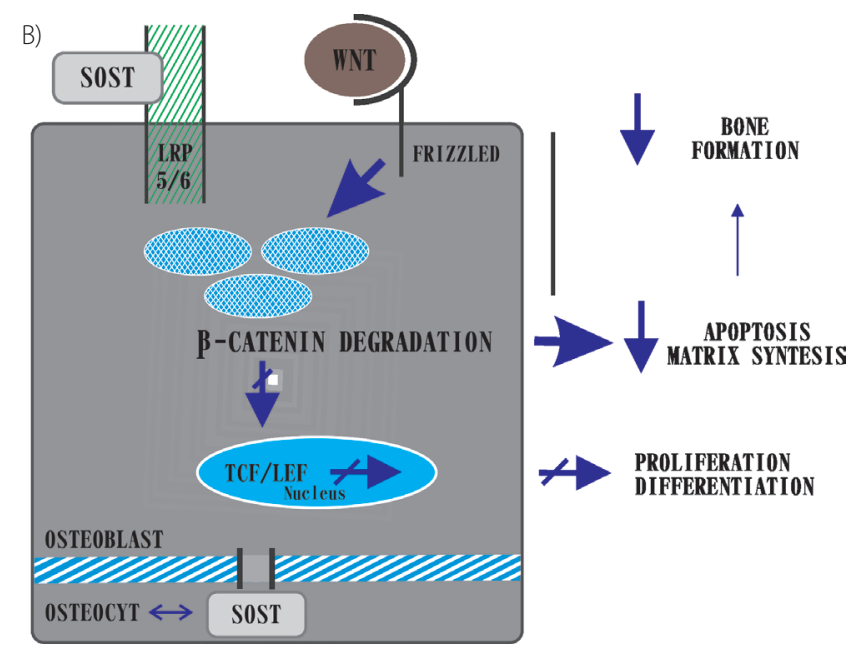

Factors indirectly exerting an inhibitory effect on the secretion of sclerostin by osteocytes are parathyroid hormone (PTH), estrogen, and mechanical stress. In turn, bone morphogenetic proteins (BMP 2,4,6), glucocorticosteroids (GKS), calcitriol (1,25-dihydroxycholecalciferol, $\left.1,25(\mathrm{OH}) \mathrm{H}_{2} \mathrm{D}_{3}\right)$ stimulate the secretion of sclerostin and thereby inhibit the activity of osteoblasts and bone formation. ${ }^{21,22}$

tically significant dose-related increases in spine, femoral neck, and total hip BMD compared with placebo. In the highest dose group, BMD increases from baseline reached $17.7 \%$ at the spine, and $6.2 \%$ at the total hip. Biochemical markers of bone formation increased rapidly during blosozumab treatment and trended toward pretreatment levels by the end of the study. These results support further study of blosozumab as a potential anabolic therapy for osteoporosis. ${ }^{69}$

For the prevention of fractures, antiresorptive drugs (bisphosphonates and denosumab) that decrease high bone resorption and, secondarily, also bone formation, are the mainstream of therapy. Osteoanabolic drugs, such as teriparatide, increase bone formation more than bone resorption and are used in severe osteoporosis, including patients treated with antiresorptive drugs in those who still lose bone and have recurrent fractures. New potential drugs for fracture prevention that uncouple bone resorption from bone formation include odanacatib, a specific inhibitor of cathepsin-K, the enzyme that degrades bone collagen type I, inhibits bone resorption and temporarily bone formation, and monoclonal antibodies against sclerostin (romosozumab, blosozumab), that stimulate bone formation and decrease bone resorption. ${ }^{70}$ Regardless of the uncertain role of sclerostin in the pathogenesis of postmenopausal osteoporosis, its neutralization is an interesting target for treatment of bone disease. McClung et al. showed that neutralization of sclerostin with a humanized monoclonal antibody (romosozumab) significantly improved bone mineral density in osteoporotic women. ${ }^{71}$ The treatment of postmenopausal women with an antibody targeted against sclerostin resulted in substantial increases in spine and hip BMD. These results are supported by study of blosozumab as a potential anabolic therapy for osteoporosis. A randomized, double-blinded, phase 2 clinical trial of blosozumab, a sclerostin antibody, in postmenopausal women with low bone mineral density. ${ }^{69}$

It has not been established how antisclerostin antibodies affect vascular calcifications. In CKD patients, osteoporosis and CKD-MBD often occur simultaneously. Blosozumab may potentially be considered as a therapeutic approach in CKD-MBD; however, the antibody dosing, efficacy, or safety in patients with CKD has yet to be determined.

\section{References}

1. Chapurlat RD, Delmas PD. Bone microdamage: A clinical perspective. Osteoporos Int. 2009;20:1299-1308.

2. Winkler DG, Sutherland MK, Geoghegan JC, et al. Osteocyte control of bone formation via sclerostin, a novel BMP antagonist. EMBO J. 2003;22:6267-6276.

3. Kramer I, Halleux $C$, Keller $\mathrm{H}$, et al. Osteocyte Wnt/\{beta\}catenin signaling is required for normal bone homeostasis. Mol Cell Biol. 2010;30:3071-3085.

4. Dallas SL, Bonewald LF. Dynamics of the transition from osteoblast to osteocyte. Ann NY Acad Sci. 2010;1192:437-443.

5. Poole KE, van Bezooijen RL, Loveridge N, et al. Sclerostin is delayed secreted product of osteocytes that inhibits bone formation. FASEB J. 2005;19:1842-1844.

6. Choi HY, Dieckmann M, Herz J, Niemeier A. Lrp4, a novel receptor for Dickkopf-1 and sclerostin, is expressed by osteoblasts and regulated bone growth and turnover in vivo. PLoS One. 2009;20:7930.

7. Li X, Zhang Y, Kang H, et al. Sclerostin binds to LRP5/6 and antagonizes canonical Wnt signaling. J Biol Chem. 2005;280:19883-19887.

8. Jackson A, Vayssière B, Garcia T, et al. Gene array analysis of Wntregulated genes in C3H10T1/2 cells. Bone. 2005;36:585-598.

9. van Oers R, van Rietbergen B, Ito K, Hilbers P, Huiskes R. A sclerostin-based theory for strain-induced bone formation. Biomech Model Mechanobiol. 2011;10:663-670.

10. Reddi AH. Initiation and promotion of endochondral bone formation by bone morphogenic proteins: Potential implications for avian tibial chondrodysplasia. Poultry Sci. 2009;79:978-981.

11. Yang F, Tang W, So S, de Crombrugghe B, Zhang C. Sclerosin is a direct target of osteoblast-specific transcription factor osterix. Biochem Biophys Res Commun. 2010;400:684-688.

12. Ott S. Sclerostin and Wnt Signaling - the pathway to bone strength. J Clin Endocrinol Metab. 2005;90:6741-6743. 
13. Krishnan V, Bryant HU, MacDougald OA. Regulation of bone mass by Wnt signaling. J Clin Invest. 2006;116:1202-1209.

14. Sutherland MK, Geoghegan JC, Yu C, et al. Sclerostin promotes the apoptosis of human osteoblastic cells: A novel regulation of bone formation. Bone. 2002;35:828-835.

15. Hill TP, Später D, Taketo MM, Birchmeier W, Hartmann C. Canonical Wnt/beta-catenin signaling prevents osteoblast from differentiating into chondrocytes. Dev Cell. 2005;8:727-738.

16. Pelletier S, Dubourg L, Carlier MC, Hadj-Aissa A, Fouque D. The relation between renal function and serum sclerostin in adult patients with CKD. Clin J Am Soc Nephrol. 2013;8:819-823.

17. Cejka D, Herberth J, Branscum AJ, et al. Sclerostin and Dickkopf-1 in renal osteodystrophy. Clin J Am Soc Nephrol. 2011;6:877-882.

18. Włodarski KH, Włodarski P. Osteoclast precursor cell fusion and regulation of nature osteoclast activity. Post Biol Kom. 2006;33:273-284.

19. Wijenayaka AR, Kogawa M, Lim HP, Bonewald LF, Findlay DM Atkins GJ. Sclerostin stimulates osteocyte support of osteoclast activity by a RANKL-dependently pathway. PLoS One. 2011;6:25900.

20. Bodine PVN. Wnt signaling control of bone cell apoptosis. Cell Research. 2008;18:248-225.

21. Khosala S, Westendorf J, Oursler M. Building bone to reverse osteoporosis and repair fractures. J Clin Invest. 2008;118:421-428.

22. Balemans $W$, Ebeling $M$, Patel $N$, et al. Increased bone density in sclerosteosis is due to the deficiency of a novel secreted protein (SOST). Hum Mol Genet. 2001;10:537-543.

23. Winkler DG, Yu C, Geoghegan JC, et al. Noggin and sclerostin bone morphogenetic protein antagonists form a mutually inhibitory complex. J Biol Chem. 2004;279:36293-36298.

24. Beighton PH, Hamersma $\mathrm{H}$, Brunkow ME. SOST-related sclerosing bone dysplasias. https://www.ncbi.nlm.nih.gov/books/NBK1228/ Accessed November 1, 2015.

25. Wergedal JE, Veskovic K, Hellan M, et al. Patients with van Buchem disease, an osteosclerotic genetic disease, have elevated bone formation markers, higher bone density, and greater derived polar moment of inertia than normal. J Clin Endocr Metab. 2003;88:5778-5783.

26. van Lierop $\mathrm{AH}, \mathrm{Ham}$ dy $\mathrm{NA}$, Hamersma $\mathrm{H}$, et al. Patients with sclerosteosis and disease carriers: Human models of the effect of sclerostin on bone turnover. J Bone Miner Res. 2011;26:2804-2811.

27. Kim SJ, Bieganski T, Sohn YB, et al. Identification of signal peptide domain SOST mutations in autosomal dominant craniodiaphyseal dysplasia. Hum Genet. 2011;129:497-502.

28. Uitterlinden AG, Arp PP, Paeper BW, et al. Polymorphisms in the sclerosteosis/van Buchem disease gene (SOST) region are associated with bone-mineral density in elderly whites. Am J Hum Genet. 2004;75:1032-1045.

29. Berdiaki A, Datsis GA, Nikitovic D, et al. Parathyroid hormone (PTH) peptides through the regulation of hyaluronam metabolism affect osteosarcoma cell migration. IUBMB Life. 2010;62:377-386.

30. Reppe S, Noer A, Grimholt RM, et al. Methylation of bone SOST, its mRNA, and serum sclerostin levels correlate strongly with fracture risk in postmenopausal women. J Bone Miner Res. 2015;30:249-256.

31. Mödder UI, Hoey KA, Amin S, et al. Relation of age, gender, and bone mass to circulating sclerostin levels in women and men. J Bone Miner Res. 2011;26:373-379.

32. Kim RY, Yang HJ, Song YM, Kim IS, Hwang SJ. Estrogen modulates bone morphogenetic protein-induced sclerostin expression through the Wnt signaling pathway. Tissue Eng Part A. 2015;21:2076-2088.

33. Mirza FS, Padhi ID, Raisz LG, Lorenzo JA. Serum sclerostin levels negatively correlate with parathyroid hormone levels and free estrogen index in postmenopausal women. J Clin Endocrinol Metab. 2010;95:1991-1997.

34. Sankaralingam A, Roplekar R, Turner C, Dalton RN, Hampson G. Changes in Dickkopf-1 (DKK1) and sclerostin following a loading dose of vitamin D2 (300,000 IU). J Osteoporos. 2014;2014:682763.

35. Rubin MR. Bone cells and bone turnover in diabetes mellitus. Curr Osteoporos Rep. 2015;13:186-191.

36. Muschitz C, Kocijan R, Marterer C, et al. Sclerostin levels and changes in bone metabolism after bariatric surgery. J Clin Endocrinol Metab. 2015;100:891-901.

37. Raisz L. Pathogenesis of osteoporosis: Concepts, conflicts, and prospects. J Clin Invest. 2005;115:3318-3325.

38. Reppe S, Noer A, Grimholt RM, et al. Methylation of bone SOST, its mRNA, and serum sclerostin levels correlate strongly with fracture risk in postmenopausal women. J Bone Miner Res. 2015;30:249-256.
39. Cheng Q, Tang W, Sheu TJ, et al. Circulating TGF- $\beta 1$ levels are negatively correlated with sclerostin levels in early postmenopausal women. Clin Chim Acta. 2016;455:87-92.

40. Ardawi MS, Al-Kadi HA, Rouzi AA, Qari MH. Determinants of serum sclerostin in healthy pre- and postmenopausal women. J Bone Miner Res. 2011;26:2812-2822.

41. Garnero P, Sornay-Rendu E, Munoz F, Borel O, Chapurlat RD. Association of serum sclerostin with bone mineral density, bone turnover, steroid and parathyroid hormones, and fracture risk in postmenopausal women: The OFELY study. Osteoporos Int. 2013;24:489-494.

42. Morales-Santana S, Díez-Pérez A, Olmos JM, et al. Circulating sclerostin and estradiol levels are associated with inadequate response to bisphosphonates in postmenopausal women with osteoporosis. Maturitas. 2015;82:402-410.

43. Ono K, Ohashi S, Tanaka S. Rheumatoid arthritis and bone - perarticular and systemic bone loss. Clin Calcium. 2013;23:249-255.

44. Charactcharoenwitthaya N, Khosla S, Atkinson EJ, McCready LK, Riggs BL. Effect of blockade of TNF- $a$ and interleukine-1 action on bone resorption in early postmenopausal women. J Bone Miner Res. 2007;22:724-729.

45. Axmann R, Böhm C, Krönke G, Zwerina J, Smolen J, Schett G. Inhibition of interleukin- 6 receptor directly blocks osteoclast formation in vitro and in vivo. Arthritis Rheum. 2009;60:2747-2756.

46. Harre $U$, Georgess D, Bang $H$, et al. Induction of osteoclastogenesis and bone loss by human autoantibodies against citrullinated vimentin. J Clin Invest. 2012;122:1791-1802.

47. Chen XX, Baum W, Dwyer D, et al. Sclerostin inhibition reverses systemic, periarticular and local bone loss in arthritis. Ann Rheum Dis. 2013;72:1732-1736.

48. Yao W, Dai W, Jiang L, et al. Sclerostin-antibody treatment of glucocorticoid-induced osteoporosis maintained bone mass and strength. Osteoporos Int. 2016;27:283-294.

49. Guañabens N, Gifre L, Peris P. The role of Wnt signaling and sclerostin in the pathogenesis of glucocorticoid-induced osteoporosis. Curr Osteoporos Rep. 2014;12: 90-97.

50. Briot K, Roux C. Glucocorticoid-induced osteoporosis. RMD Open. 2015;8:1,e000014.

51. Koos R, Brandenburg V, Mahnken AH, et al. Sclerostin as a potential novel biomarker for aortic valve calcification: An in vivo and ex vivo study. J Heart Valve Dis. 2013;22:317-325.

52. Hampson G, Edwards S, Conroy S, Blake GM, Fogelman I, Frost ML. The relationship between inhibitors of the Wnt signalling pathway (Dickkopf-1(DKK1) and sclerostin), bone mineral density, vascular calcification and arterial stiffness in post-menopausal women. Bone. 2013;56:42-47.

53. Kuipers AL, Miljkovic I, Carr JJ, et al. Association of circulating sclerostin with vascular calcification in Afro-Caribbean men. Atherosclerosis. 2015;239:218-223.

54. Quarles LD. Role of FGF23 in vitamin D and phosphate metabolism: Implications in chronic kidney disease. Exp Cell Res. 2012;15:1040-1048.

55. Jean G, Mayor B, Deleaval P, et al. Vascular calcification progression is an independent predictor of mortality in patients on haemodialysis. Nephron. 2015;130:169-174.

56. London G, Guerin A, Marchais S, Métivier F, Pannier B, Adda H. Arterial media calcification in end-stage renal disease: Impact on all-cause and cardiovascular mortality. Nephrol Dial Transplant. 2003;18:1731-1740.

57. de Oca A, Maduen J, Martinez-Moreno J, et al. High-phosphateinduced calcification is related to SM22a promoter methylation in vascular smooth muscle cells. J Bone Mineral Res. 2010;25:19962005.

58. Franceschi RT, Xiao G. Regulation of the osteoblast-specific transcription factor, Runx2: Responsiveness to multiple signal transduction pathways. J Cell Biochem. 2003;88:446-454.

59. Zhu D, Mackenzie NC, Millán JL, Farquharson C, MacRae VE. The appearance and modulation of osteocyte marker expression during calcification of vascular smooth muscle cells. PLoS One. 2011;6:19595.

60. Unver S, Kavlak E, Gümüsel HK, et al. Correlation between hypervolemia, left ventricular hypertrophy and fibroblast growth factor 23 in hemodialysis patients. Ren Fail. 2015;37:951-956.

61. Drechsler C, Evenepoel P, Vervloet MG, et al. High levels of circulating sclerostin are associated with better cardiovascular surviv- 
al in incident dialysis patients: Results from the NECOSAD study. Nephrol Dial Transplant. 2015;30:288-293.

62. Delanaye P1, Krzesinski JM, Warling X, et al. Clinical and biological determinants of sclerostin plasma concentration in hemodialysis patients. Nephron Clin Pract. 2014; 128:127-134

63. Morena M, Jaussent I, Dupuy AM, et al. Osteoprotegerin and sclerostin in chronic kidney disease prior to dialysis: Potential partners in vascular calcifications. Nephrol Dial Transplant. 2015;30:1345-1356.

64. Cejka D, Marculescu R, Kozakowski N, et al. Renal elimination of sclerostin increases with declining kidney function. J Clin Endocrinol Metab. 2014;99:248-255.

65. Kanbay M, Siriopol D, Saglam M, et al. Serum sclerostin and adverse outcomes in nondialyzed chronic kidney disease patients. J Clin Endocrinol Metab. 2014;99:1854-1861.

66. Asamiya Y, Yajima A, Shimizu S, Otsubo S, Tsuchiya K, Nitta K. Associations between the levels of sclerostin, phosphate, and fibroblast growth factor-23 and treatment with vitamin $D$ in hemodialysis patients with low intact PTH level. Osteoporos Int. 2015;26:1017-1028.

67. Ishimura E, Okuno S, Ichii M, et al. Relationship between serum sclerostin, bone metabolism markers and bone mineral density in maintenance hemodialysis patients. J Clin Endocrinol Metab. 2014;99:4315-4320.

68. Fang $Y$, Ginsberg $C$, Sugatani T, Monier-Faugere MC, Malluche $\mathrm{H}_{\text {, }}$ Hruska KA. Early chronic kidney disease-mineral bone disorder stimulates vascular calcification. Kidney Int. 2014;85:142-150.

69. Recker RR, Benson CT, Matsumoto T, et al. A randomized, doubleblind phase 2 clinical trial of blosozumab, a sclerostin antibody, in postmenopausal women with low bone mineral density. J Bone Miner Res. 2015;30:216-224.

70. Geusens P. New insights into treatment of osteoporosis in postmenopausal women. RMD Open. 2015;15:1,e000051.

71. McClung MR, Grauer A, Boonen S, et al. Romosozumab in postmenopausal women with low bone mineral density. N Engl J Med. 2014;370:412-420. 